\title{
Possible Prosthetic Valve Endocarditis by Pandoraea pnomenusa and Specific Virulence Mechanisms
}

This article was published in the following Dove Press journal:

Infection and Drug Resistance

\author{
Elias Bodendoerfer ${ }^{\prime}$ \\ Nicolas Personnic' \\ Carlos A Mestres ${ }^{2}$ \\ Markus J Wilhelm² \\ Lilly Meyer ${ }^{3}$ \\ Barbara Hasse ${ }^{3}$
}

'University of Zurich, Institute of Medical Microbiology, Zurich, Switzerland; ${ }^{2}$ University Hospital Zurich, Clinic for Cardiac Surgery, Zurich, Switzerland; ${ }^{3}$ University Hospital Zurich, Department of Infectious Diseases and Hospital Epidemiology, Zurich, Switzerland
Correspondence: Elias Bodendoerfer Institute of Medical Microbiology, University of Zurich, Gloriastrasse 28/30,

Zürich, $\mathrm{CH}-8006$, Switzerland

Tel +4I 4463426 I8

Email ebodendoerfer@imm.uzh.ch

\begin{abstract}
Bacteremia by Pandoraea spp. has rarely been described before. We report the first case of a P. pnomenusa possible prosthetic valve endocarditis, according to the modified Duke criteria, in a 37-year old male injecting drug user suffering from recurrent endocarditis. Furthermore, we demonstrate biofilm formation by the $P$. pnomenusa isolates of this patient and investigate antibiotic resistance.

Keywords: prosthetic valve endocarditis, central venous catheter, bacteremia, Pandoraea pnomenusa, biofilm formation, antibiotic resistance
\end{abstract}

\section{Introduction}

The genus Pandoraea was first described in $2000^{1}$ and is mostly isolated from individuals with cystic fibrosis $(\mathrm{CF})$. The genus currently contains 28 validly published taxa, ${ }^{2,3}$ with more being added continuously. ${ }^{4}$ Although the true prevalence is unknown, it seems to be a rare organism. ${ }^{5}$ Current evidence suggests a pathogenic role of Pandoraea spp. in chronic lung infection with accelerated decline of lung function. ${ }^{5}$ Biofilm formation is a key virulence factor associated with colonization and infection in CF lungs. In addition, biofilm formation can lead to antibiotic resistance and persistence of various pathogens in infected lungs. ${ }^{5-8}$

Currently, biofilm formation is insufficiently studied in Pandoraea spp, and little is known about the virulence factors of Pandoraea pnomenusa. ${ }^{9,10}$ Several cases of bloodstream infections (BSI) with Pandoraea spp. have been described $^{11-15}$ and thus far, only two cases of $P$. pnomenusa bacteremia have been reported. $^{12,13}$ We aim at reporting the possible case of $P$. pnomenusa prosthetic valve endocarditis (PVE) in a patient suffering from recurrent endocarditis. We also investigated biofilm formation and antibiotic resistance determinants.

\section{Case Presentation}

A 37-year old male injecting drug user (IDU) with recurrent biological PVE resided in a cardiac rehabilitation clinic after valve re-replacement surgery for an episode of Enterococcus faecalis and Candida dubliniensis endocarditis. Two days after completion of his six-week antibiotic treatment with intravenous amoxicillin/clavulanic acid and whilst still under isavuconazole treatment, he developed fever accompanied by elevated inflammatory markers. Subsequently, two blood culture bottles yielded growth of $P$. pnomenusa drawn simultaneously 
from his peripherally inserted central venous catheter (PICC) and from his elbow vein. The patient was started on empirical treatment with tigecycline. Upon suspicion of a central venous catheter-associated bloodstream infection (CLABSI), the patient was readmitted to the University Hospital of Zurich on day 6 of the current episode. Blood cultures drawn upon admission showed a marked difference in time to positivity of $9 \mathrm{~h}$ between the PICC (14 hours) and the peripherally drawn blood cultures (23 hours), indicative of CLABSI. The central line was removed immediately without showing any growth microbiologically. Initial testing demonstrated susceptibility towards piperacillin/tazobactam but resistance to tigecycline when interpreted according to Pseudomonas clinical breakpoints. Accordingly, therapy was switched to piperacillin/tazobactam on day 8 of the current episode. Three days after initiation of targeted therapy, a new PICC was inserted until novel blood cultures on day 22 yielded growth of $P$. pnomenusa again, which led to another change of the PICC. At the same time, transesophageal echocardiography (TOE) demonstrated filiform, floating structures on the central catheter without valve vegetation, whereas the positron emission computed tomography (PET/CT) did neither show a metabolic activity of the prosthetic valve nor of the catheter tip. A small echo-dense structure at the anterior pocket of the annulus had already been described in earlier investigations. Based on suggestive clinical and microbiological criteria (fever, persistent positive blood cultures not meeting major criteria, predisposing heart condition and IDU $^{16}$ ) a treatment duration of six weeks for possible PVE was determined. After a three-week course of piperacillin/tazobactam, therapy was switched to trimethoprim/sulfamethoxazole. To date, $P$. pnomenusa was not isolated in the patient again.

\section{Methods}

The BacT/ALERT Virtuo blood culture system was used for incubation (bioMérieux (Suisse) SA,1213 Petit Lancy).

Antimicrobial susceptibility testing was performed according to EUCAST. ${ }^{17}$ Minimal inhibitory concentrations (MIC) were determined by ETEST (bioMérieux (Suisse) SA, 1213 Petit Lancy, Switzerland) or MIC strip test (Liofilchem srl, Roseto degli Abruzzi, Italy). Additionally, the Rapidec Carba NP test was performed to confirm carbapenemase activity (bioMérieux
(Suisse) SA, 1213 Petit Lancy, Switzerland). Klebsiella pneumoniae ATCC BAA-2146 was used as positive control strain.

We evaluated quantitative biofilm formation according to O'Toole. ${ }^{18}$ Colonies of Pandoraea spp. were suspended in LB Broth and grown overnight at $37^{\circ} \mathrm{C}$, crystal violet was solubilized with $96 \%$ ethanol and optical density (OD) was measured at $620 \mathrm{~nm}$. In parallel, the bacterial suspensions were seeded in imaging chambers (ibidi) and grown to form biofilms at $37^{\circ} \mathrm{C}$, as described previously. ${ }^{19}$ After fixation with $4 \%$ paraformaldehyde (Electron Microscopy Sciences), biofilms were stained with $\mathrm{FM}^{\circledR}$ 4-64FX (Molecular Probes) and analyzed by confocal microscopy (excitation $561 \mathrm{~nm}$, emission 600-620 nm). Image processing was performed with ImageJ software (https://imagej. net/Fiji).

For whole-genome sequencing (WGS) DNA libraries were prepared following the QIAseq FX DNA Library Kit (QIAGEN AG, Hombrechtikon, Switzerland). Quality control of the library was performed using capillary electrophoresis (Fragment Analyzer Automated CE System, Advanced Analytical). Sequencing was done on the MiSeq Platform (Illumina). The Comprehensive Antibiotic Resistance Database (CARD) was used to identify resistance genes. ${ }^{20}$

\section{Microbiological Results}

Initial $16 \mathrm{~S}$ sequencing did not yield reliable results. Thus, species identification was done by whole genome sequencing and the isolate was identified as $P$. pnomenusa. ${ }^{3}$, (Supplementary Figure 1, We identified an OXA-151 carbapenemase with two amino acid replacements V180L and S271A.21 The isolates were highly resistant to meropenem but susceptible to imipenem. The Rapidec Carba NP test yielded no perceivable hydrolysis of imipenem. The susceptibility of piperacillin/tazobactam was surprising (Table 1).

We assessed the ability of the clinical $P$. pnomenusa isolates to produce static biofilms on abiotic surfaces. As controls, we included the bacterial species Pseudomonas aeruginosa and Klebsiella pneumoniae. $18 \mathrm{~h}$ biofilms were stained with $\mathrm{FM}^{\circledR} 4-64 \mathrm{FX}$ to label the bacteria and immediately examined by confocal microscopy. Analysis revealed that all $P$. pnomenusa isolates, as well as the control species, formed three-dimensional structures in a rich medium with irregular and fluffy aggregates (Figure 1). These aggregates formed in some areas of the surface, while in other areas single 
Table I Susceptibility Testing of the $P$. pnomenusa Strains

\begin{tabular}{|c|c|c|c|c|c|c|}
\hline Antibiotic & $\begin{array}{l}\text { Strain I } \\
{[\mathrm{mm}]}\end{array}$ & $\begin{array}{l}\text { Strain I [mg/ } \\
\text { L] }\end{array}$ & Interpretation & $\begin{array}{l}\text { Strain } 2 \\
{[\mathrm{~mm}]}\end{array}$ & $\begin{array}{l}\text { Strain } 2[\mathrm{mg} / \\
\text { L] }\end{array}$ & Interpretation \\
\hline Piperacillin & & 24 & $\mathrm{R}$ & & 8 & $\mathrm{R}$ \\
\hline Piperacillin/tazobactam & 23 & 0.19 & S & 24 & 0.047 & S \\
\hline Ceftazidime & 6 & 24 & $\mathrm{R}$ & 6 & 16 & $\mathrm{R}$ \\
\hline Ceftazidime/avibactam & & 24 & $\mathrm{R}$ & & 24 & $R$ \\
\hline Cefepime & 26 & & S & 27 & & S \\
\hline Imipenem & 29 & 1.5 & $S$ & 27 & 1.5 & S \\
\hline Meropenem & 6 & $\geq 32$ & $\mathrm{R}$ & 6 & $\geq 32$ & $\mathrm{R}$ \\
\hline Meropenem/vaborbactam & & 32.0 & $\mathrm{R}$ & & 24 & $\mathrm{R}$ \\
\hline Ciprofloxacin & 28 & 0.5 & S & 28 & 0.5 & S \\
\hline Levofloxacin & 28 & & S & 30 & & $S$ \\
\hline $\begin{array}{l}\text { Trimethoprim/ } \\
\text { sulfamethoxazole }\end{array}$ & 36 & 0.64 & $S$ & 33 & 0.64 & S \\
\hline Amikacin & 14 & & $\mathrm{R}$ & 9 & & $\mathrm{R}$ \\
\hline Gentamicin & 6 & & $\mathrm{R}$ & 6 & & $\mathrm{R}$ \\
\hline Tobramycin & 11 & & $\mathrm{R}$ & 6 & & $\mathrm{R}$ \\
\hline Ceftolozane/tazobactam & & $\leq 0.016$ & S & & & \\
\hline Tetracyclin & & 3.0 & & & & \\
\hline Minocycline & & 0.38 & & & & \\
\hline Tigecycline & & 1.5 & $\mathrm{R}$ & & & \\
\hline
\end{tabular}

Note: For disc diffusion testing EUCAST breakpoints for P. aeruginosa were applied.

cells attached to the surface. Biofilm formation on abiotic surfaces can be quantified by crystal violet staining of the biomass attached to the surfaces of 96-well plates. Under the conditions tested, absorbance at $620 \mathrm{~nm}$ of P. pnomenusa isolates 1 and 2 were 0.42 and 0.60 , respectively (Figure 2). Compared to the control strains, $P$. pnomenusa produced comparable amounts of biofilmassociated biomass.

\section{Discussion}

We report the case of a possible PVE due to the uncommon biofilm-producing pathogen $P$. pnomenusa.

Pandoraea spp. is an emerging pathogen in CF patients. ${ }^{5}$ Rarely BSI are reported in patients with other comorbidities. ${ }^{11-15}$ A fatal case of $P$. pnomenusa BSI was reported in a 30-year old patient suffering from end-stage pulmonary sarcoidosis with secondary nocardiosis and mycetomas. $^{12}$ The second published case of $P$. pnomenusa BSI occurred in 10-month old child with acute lymphoblastic leukemia that was successfully treated with imipenem and removal of the port-a-cath system. $^{13}$ BSI with Pandoraea spp. other than $P$. pnomenusa were reported in immunocompromised patients after lung, ${ }^{14}$ liver transplantation ${ }^{11}$ and cystic fibrosis. ${ }^{15}$
To date a possible PVE due to Pandoraea spp. has not been reported. Certain elements of this case suggest a possible endocarditis, ${ }^{16}$ whereby neither the TOE nor the PET/CT could ultimately confirm the diagnosis. The patient was treated with piperacillin/tazobactam although piperacillin/tazobactam was reported to be resistant in Pandoraea spp thus far. ${ }^{22}$ Pandoraea spp. are known to harbor OXA family carbapenemases, and OXA-62-like carbapenemases were described in $P$. pnomenusa. $^{22}$ However, we identified an OXA-151 carbapenemase with two amino acid replacements V180L and S271A. Interestingly, these AA "replacements" are found in OXA-62, while L18P, A33T and T245A are discordant from OXA-62 but found in OXA-151. ${ }^{22}$ Nonetheless, the strain was highly resistant to meropenem but susceptible to imipenem.

We demonstrated that $P$. pnomenusa is capable of forming biofilms. Thus far, this had been demonstrated solely for Pandoraea pulmonicola. ${ }^{9}$ For many pathogens, biofilm formation contributes to virulence in $\mathrm{CF}$ lungs. ${ }^{8}$ Arguably, biofilm formation also facilitates the colonization of intravenous catheters and promotes endocarditis. ${ }^{8,16}$ With this case report, we hope to expand the current understanding of Pandoraea spp. but further research is warranted, especially to prove a causal link 

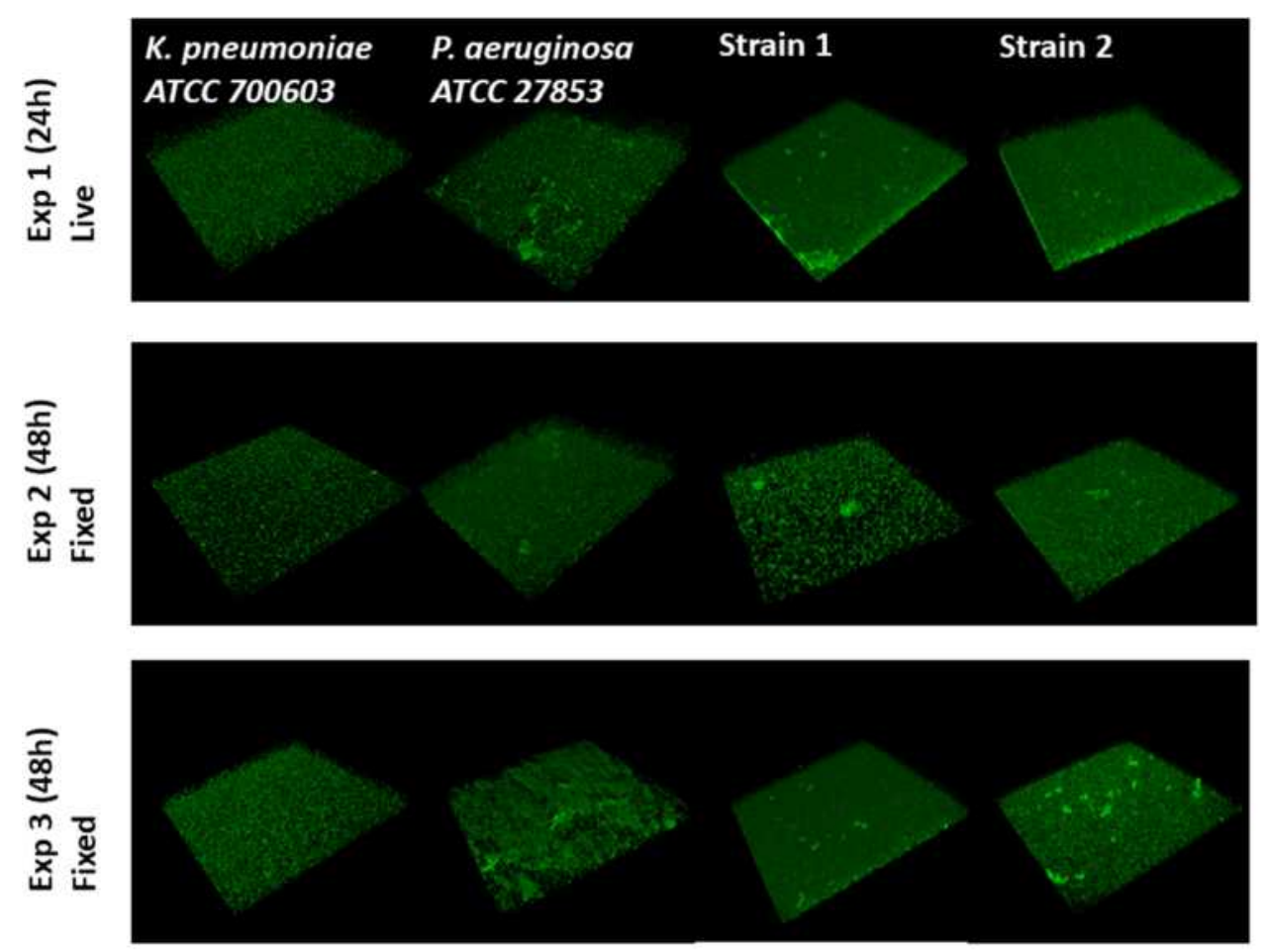

$\mathrm{Y}=260 \mu \mathrm{m} ; \mathrm{X}=260 \mu \mathrm{m} ; \mathrm{Z}=30 \mu \mathrm{m}$

Figure I Pandoraea pnomenusa forms biofilm on abiotic surfaces. Overnight fully aerated planktonic culture of $P$. pnomenusa patient isolates (strains \#I and \#2) as well as the biofilm-producing species K. pneumoniae ATCC 700603 and P. aeruginosa ATCC 27853 (ie, positive controls) were diluted I: 100 in fresh LB medium and grown at $37^{\circ} \mathrm{C}$, under agitation, to exponential phase. Cultures were then inoculated into tissue culture treated imaging chambers and let form biofilms at $37^{\circ} \mathrm{C}$. At given time-points, biofilms were washed using PBS to remove non-sessile bacteria and, when indicated, fixed with $4 \%$ paraformaldehyde. Bacterial membranes were subsequently stained with the fluorescent probes FM 4-64FX to visualise the biofilm biomass by confocal laser scanning microscopy. 3D reconstructions of the $Z$ optical section stacks $(x=260 \mu \mathrm{m}, y$ $=260 \mu \mathrm{m}, z=30 \mu \mathrm{m})$ indicates the formation of dense and rough biofilms of distinct morphotypes. $K$. pneumoniae produced small, uniformly distributed aggregates. $P$. aeruginosa and $P$. pnomenusa developed large aggregates, distributed across the whole well.

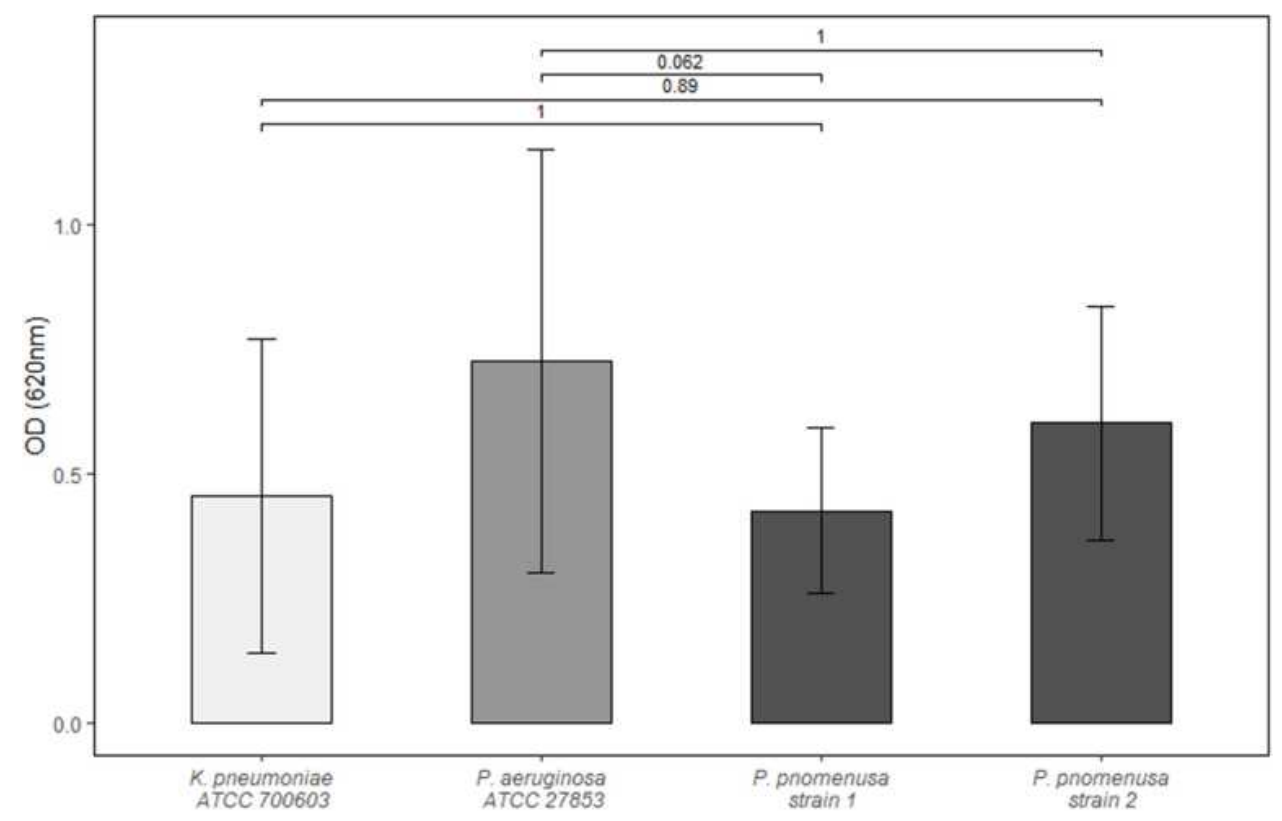

Figure 2 Quantitative biofilm assay. Blank wells containing Müller-Hinton-broth were used to determine background optical density (OD). Each bar represents the arithmetic mean of independent triplicates with at least four measurements each. Standard deviation is depicted as whiskers. Represented above are the Bonferroni adjusted p-values obtained by $t$-test. 
between biofilm formation by Pandoraea spp. and virulence in CF patients, CLABSI and endocarditis.

\section{Sequence Information}

Sequences are available at the European nucleotide archive.

Strain 1ERS5743847

Strain 2ERS5743848

\section{Ethical Approval}

Ethical approval was not required to publish the case details.

\section{Informed Consent}

The patient has given written permission to publish this case report.

\section{Acknowledgments}

We thank first and foremost the patient, as well as all our co-workers at the Institute of Medical Microbiology of the University of Zurich and the University Hospital of Zurich.

\section{Disclosure}

The authors report no conflicts of interest in this work.

\section{References}

1. Coenye T, Falsen E, Hoste B, et al. Description of Pandoraea gen. nov. with Pandoraea apista sp. nov., Pandoraea pulmonicola sp. nov., Pandoraea pnomenusa sp. nov., Pandoraea sputorum sp. nov. and Pandoraea norimbergensis comb. nov. Int J Syst Evol Microbiol. 2000;50(Pt 2). doi:10.1099/00207713-50-2-887

2. Peeters C, Canck E, De, Cnockaert M, et al. Comparative genomics of Pandoraea, a genus enriched in xenobiotic biodegradation and metabolism. Front Microbiol. 2019;10:2556. doi:10.3389/fmicb.20 19.02556

3. Parte AC, Sardà Carbasse J, Meier-Kolthoff JP, Reimer LC, Göker M. List of Prokaryotic names with Standing in Nomenclature (LPSN) moves to the DSMZ. Int $J$ Syst Evol Microbiol. 2020;70 (11):5607-5612. doi:10.1099/ijsem.0.004332

4. Kostygov AY, Butenko A, Nenarokova A, et al. Genome of Ca. Pandoraea novymonadis, an endosymbiotic bacterium of the trypanosomatid Novymonas esmeraldas. Front Microbiol. 2017;8:1940. doi:10.3389/fmicb.2017.01940

5. Green H, Jones AM. Emerging gram-negative bacteria: pathogenic or innocent bystanders. Curr Opin Pulm Med. 2018;24(6):592-598. doi:10.1097/MCP.0000000000000517

6. Green H, Jones AM. The microbiome and emerging pathogens in cystic fibrosis and non-cystic fibrosis bronchiectasis. Semin Respir Crit Care Med. 2015;36(2):225-235. doi:10.1055/s-0035-1546752
7. Høiby N, Ciofu O, Bjarnsholt T. Pseudomonas aeruginosa biofilms in cystic fibrosis. Future Microbiol. 2010;5(11):1663-1674. doi: $10.2217 / \mathrm{fmb} .10 .125$

8. Gupta P, Sarkar S, Das B, Bhattacharjee S, Tribedi P. Biofilm, pathogenesis and prevention-a journey to break the wall: a review. Arch Microbiol. 2016;198(1):1-15. doi:10.1007/s00203-015-1148-6

9. Caraher E, Collins J, Herbert G, et al. Evaluation of in vitro virulence characteristics of the genus Pandoraea in lung epithelial cells. $J$ Med Microbiol. 2008;57(1):15-20. doi:10.1099/jmm.0.47544-0

10. Costello A, Herbert G, Fabunmi L, et al. Virulence of an emerging respiratory pathogen, genus Pandoraea, in vivo and its interactions with lung epithelial cells. J Med Microbiol. 2011;60(3):289-299. doi:10.1099/jmm.0.022657-0

11. Xiao X, Tian H, Cheng X, et al. Pandoraea sputorum bacteremia in a patient who had undergone allogeneic liver transplantation plus immunosuppressive therapy: a Case Report. Infect Drug Resist. 2019;12:3359-3364. doi:10.2147/IDR.S227643

12. Stryjewski ME, LiPuma JJ, Messier RH, Reller LB, Alexander BD. Sepsis, multiple organ failure, and death due to Pandoraea pnomenusa infection after lung transplantation. J Clin Microbiol. 2003;41 (5):2255-2257. doi:10.1128/jcm.41.5.2255-2257.2003

13. Falces-Romero I, Gutiérrez-Arroyo A, Romero-Gómez MP. Catheterassociated bacteremia by Pandoraea pnomenusa in an infant with acute lymphoblastic leukaemia. Med Clin (Engl Ed). 2016;147 (3):132. doi:10.1016/j.medcle.2016.09.017

14. Pimentel JD, MacLeod C. Misidentification of Pandoraea sputorum isolated from sputum of a patient with cystic fibrosis and review of Pandoraea species infections in transplant patients. J Clin Microbiol. 2008;46(9):3165-3168. doi:10.1128/JCM.00855-08

15. Johnson LN, Han J-Y, Moskowitz SM, Burns JL, Qin X, Englund JA. Pandoraea bacteremia in a cystic fibrosis patient with associated systemic illness. Pediatr Infect Dis J. 2004;23(9):881-882. doi:10.1097/01.inf.0000136857.74561.3c

16. Habib G, Lancellotti P, Antunes MJ, et al. 2015 ESC Guidelines for the management of infective endocarditis. The task force for the management of infective endocarditis of the European Society of Cardiology (ESC) endorsed by: European Association for Cardio-Thoracic Surgery (EACTS), the European Association of Nuclear Medicine (EANM). Eur Heart J. 2015;36(44):3075-3128. doi:10.1093/eurheartj/ehv319

17. The European Committee on Antimicrobial Susceptibility Testing. Breakpoint tables for interpretation of MICs and zone diameters.: version 9.0, valid from 2019-01-01; 2019.

18. O'Toole GA. Microtiter dish biofilm formation assay. Journal of Visualized Experiments: JoVE. 2011;(47):e2437. doi:10.3791/2437

19. Personnic N, Striednig B, Hilbi H. Quorum sensing controls persistence, resuscitation, and virulence of Legionella subpopulations in biofilms. ISME J. 2020;15(1):196-210. doi:10.1038/s41396-02000774-0

20. Jia B, Raphenya AR, Alcock B, et al. CARD 2017: expansion and model-centric curation of the comprehensive antibiotic resistance database. Nucleic Acids Res. 2017;45(D1):D566-D573. doi:10. 1093/nar/gkw1004

21. Schneider I, Queenan AM, Bauernfeind A. Novel carbapenem-hydrolyzing oxacillinase OXA-62 from Pandoraea pnomenusa. Antimicrob Agents Chemother. 2006;50(4):1330-1335. doi:10.1128/AAC.50.4.1330-1335.2006

22. Schneider I, Bauernfeind A. Intrinsic carbapenem-hydrolyzing oxacillinases from members of the genus Pandoraea. Antimicrob Agents Chemother. 2015;59(11):7136-7141. doi:10.1128/AAC.01112-15 


\section{Publish your work in this journal}

Infection and Drug Resistance is an international, peer-reviewed openaccess journal that focuses on the optimal treatment of infection (bacterial, fungal and viral) and the development and institution of preventive strategies to minimize the development and spread of resistance. The journal is specifically concerned with the epidemiology of antibiotic resistance and the mechanisms of resistance development and diffusion in both hospitals and the community. The manuscript management system is completely online and includes a very quick and fair peerreview system, which is all easy to use. Visit http://www.dovepress.com/ testimonials.php to read real quotes from published authors. 